\title{
Optimal source placement for sound zone reproduction with first order reflections $^{\mathrm{a})}$
}

\author{
Marek Olik, ${ }^{\text {b) }}$ Philip J. B. Jackson, and Philip Coleman \\ Centre for Vision, \\ Speech and Signal Processing, \\ University of Surrey, \\ Guildford, \\ Surrey, \\ GU2 7XH, \\ United Kingdom \\ Jan Abildgaard Pedersenc) \\ Bang \& Olufsen A/S, \\ Peter Bangs Vej 15, \\ DK7600, \\ Struer, \\ Denmark
}




\begin{abstract}
The problem of delivering personal audio content to listeners sharing the same acoustic space has recently attracted attention. It has been shown that a perceptually acceptable level of acoustic separation between the listening zones is difficult to achieve with active control in non-anechoic conditions. A common problem of strong first order reflections has not been examined in detail for systems with practical constraints. Acoustic contrast maximization combined with optimization of source positions is identified as a potentially effective control strategy when strong individual reflections occur. An analytic study is carried out to describe the relationship between the performance of a $2 \times 2$ (two sources and two control sensors) system and its geometry in a single-reflection scenario. The expression for acoustic contrast is used to formulate guidelines for optimizing source positions, based on three distinct techniques: Null-Split, Far-Align, and Near-Align. The applicability of the techniques to larger systems with up to two reflections is demonstrated using numerical optimization. Simulation results show that optimized systems produce higher acoustic contrast than non-optimized source arrangements and an alternative method for reducing the impact of reflections (sound power minimization).
\end{abstract}

PACS numbers: 43.60.Fg, 43.55.Jz, 43.38.Hz, 43.60.Pt 


\section{Introduction}

The problem of sound zones arises from the desire to reproduce different audio material for listeners occupying the same acoustic space. It is possible to create independent listening regions using an array of loudspeakers and active control of sound ${ }^{1}$. Such a personal audio system offers a useful alternative to headphones, which may be uncomfortable when worn over long periods, impede conversation, and hinder audibility of sounds from the environment.

The main requirement for a sound zone system is to produce large acoustic separation (acoustic contrast) between two or more regions in space by attenuating the audio played for one listener in locations occupied by the others. In sound zone nomenclature, each listener occupies the acoustically bright zone for their own audio program while remaining in the dark zone for other programs. The minimum acoustic contrast required for the listener to find the interference from the unwanted program acceptable depends on the program type and the listening circumstances, and may be in the range of $10-40 \mathrm{~dB}^{2}$. The capability of a system to produce such contrast may depend on parameters such as the number of sources and control sensors, their geometry, sound zone size and relative position, control method, and the acoustic environment. Experimental results reported in the literature show that the lower bounds of the required contrast are attainable and can be exceeded by most physical systems, irrespective of their specification or acoustic conditions ${ }^{3-12}$. The upper limit has only been approached in an anechoic or a strongly damped room $^{8,9}$, or with the zones located close to the sources ${ }^{4,10}$ where the direct-to-reverberant energy ratio (DRR) is large. This indicates the damaging effect of room reflections on contrast. The amount of contrast deterioration

a) Portions of this work were presented in M. Olik, P. J. B. Jackson, and P. Coleman, "Influence of low-order room reflections on sound zone system performance," in Proceedings of Meetings on Acoustics, Vol. 19, 2013. Presented at ICA 2013, Montreal, 2-7 June 2013.

b) Electronic address: Electronicaddress :m.olik@surrey.ac.uk

c) Present address: Dynaudio A/S, Sverigesvej 15, DK8660, Skanderborg, Denmark. 
due to reflections has been quantified in studies that evaluated the same systems both under anechoic and reflective conditions ${ }^{4,9,10,13}$. These results are summarized in Table I, showing that the lowest contrast decreased by at least $5 \mathrm{~dB}$ when reflections occurred. For systems just reaching the required minimum contrast under anechoic conditions, such a change may render interference from the competing sound program perceptually unacceptable.

Methods for reducing the impact of reflections on the performance of sound zone systems, that are complementary to passive acoustic treatment or locating the sources close to zones, have also been investigated. Elliott et al. ${ }^{13}$ proposed regularization as a means for improving contrast in a diffuse sound field. Simón-Gálvez et al. ${ }^{11}$ used loudspeakers with hyper-cardioid directivity to reduce radiation to the back of the array, potentially diminishing the influence of reflections from the wall behind. Wen et al. ${ }^{14}$ showed the advantages of using the optimal beamformer with a constrained control effort (maximum control gain method, also referred to as brightness control $(\mathrm{BC})^{1,15}$, or control effort minimization ${ }^{5}$ ) in a reflective room. The problem of limiting the influence of indirect sound on performance has not be discussed explicitly in other sound zone studies, but techniques that can reduce the array radiation similarly to regularization, directive sources, or effort-constrained beamforming have been discussed. These included the sound power minimization (SPM) method ${ }^{5,16}$ and surrounding the bright zone with a two-layered source array ${ }^{17}$. In a number of studies, room responses were incorporated into the source weight optimization process, which resulted in active attenuation of reflections in the dark zone $\mathrm{z}^{4,9,10,12}$.

A detailed evaluation of the methods listed above has not been carried out in reflective rooms. Selecting a sound zone reproduction strategy that is best suited for a particular system and type of room is therefore an unsolved problem. In commercial systems for sound reproduction in the home it is often desirable to reduce the system size, as rooms are not used exclusively for listening. Acoustic studies on domestic rooms show typically low reverberation time and uneven distribution of absorptive materials such as furniture and decorative elements ${ }^{18}$. In such rooms the sound field is far from diffuse and can be dominated by the direct sound and specular first order reflections ${ }^{19}$ (for instance from exposed walls) 
that affect sound reproduction when uncontrolled ${ }^{20}$. Furthermore, contrary to headrest systems ${ }^{4,5}$, locating loudspeakers close to the listener is usually impractical. Motivated by such applications, this article focuses on determining the most suitable control strategy for small domestic sound zone systems with strong individual room reflections and developing techniques for increasing the contrast with loudspeakers displaced from the listener.

First, sound zone generation strategies that can be effective with small systems are considered (Sec. II). Combining the acoustic contrast control (ACC) method $^{15}$ with optimization of source positions is identified as a means of maximizing contrast when strong isolated reflections occur. The optimization problem is examined systematically, starting with the analysis of a $2 \times 2$ system (two monopole sources and two control sensors) with a single surface (Sec. III). The expression for acoustic contrast produced by the system is derived, providing a common framework for exploring directivity optimization and active attenuation of a reflection. The analysis (Sec. IV) results in three techniques to reduce indirect sound in the dark zone: Null-Split (pointing the directivity null at the surface and exploiting the array symmetry), Far-Align (null sharing between the dark zone and surface) and Near-Align (taking advantage of the spatial match between the sources and their images with respect to the dark zone). The geometrical requirements for improved direct sound control discussed in the literature ${ }^{4,5}$ are also formalized and put on a common algebraic footing with the Null-Split, Far-Align, and Near-Align techniques. Furthermore, acoustical simulations are used to formulate guidelines for selecting the most suitable technique for a given system-surface geometry (Sec. V). Source positions are also optimized numerically for the $2 \times 2$ and extended systems $(2 \times 50$ and $3 \times 50)$ with up to two surfaces, and the obtained geometries are related to the analytic solutions. The optimized systems are evaluated for acoustic contrast, and the results are compared with those of non-optimized configurations, an alternative reflection strategy (SPM), and the upper contrast limit produced by the system optimized for and performing under anechoic conditions. Finally, the robustness of the techniques to implementation errors is examined and the conclusions are drawn (Sec. VI). 


\section{Background}

In this section, aspects of direct sound and reflection control with small systems are discussed and methods for improving performance are identified, followed by the introduction of a $2 \times 2$ sound zone system. The acoustic contrast measure and the ACC method are also described.

\section{A. Identification of a suitable control strategy}

In the considered acoustic environment, a sound zone system must be able to attenuate the direct sound and low order reflections effectively in the dark zone, while directing sound energy into the bright zone to produce large contrast. The aforementioned ACC method has been shown to provide large contrast over a wider bandwidth than least squares pressure matching ${ }^{10,11,21}(\mathrm{PM})$ with a limited number of sources under anechoic conditions ${ }^{1}$. Furthermore, it was demonstrated for small systems operating in a free field that ACC can outperform SPM or $\mathrm{BC}^{5}$. The effectiveness of $\mathrm{ACC}$ in controlling the direct sound will depend, among other things, on source positioning. With a limited number of control sensors in commercial systems, a compact source arrangement may be beneficial. This was demonstrated by Elliott and Jones ${ }^{4}$ who examined a pair of free-field monopoles, arranged to simulate a personal audio system for listeners in two adjacent aircraft seats. Compared to a widely-spaced array, compact sources resulted in a broader directivity null that encompassed all of the dark zone sensors, thus improving contrast. The above discussion warrants the choice of ACC based on a compact source array as the most suitable approach for the considered problem, provided that appropriate handling of reflections is ensured.

In the presence of a strong reflection, locating the source on the reflecting surface avoids interference from image sources, but restricts the array geometry and may not always be feasible. An alternative approach could be to use regularization to keep the array effort below a certain limit ${ }^{1}$. This would reduce the radiation from the array at problematic frequencies, increasing the system's robustness to reflections. However, contrast achieved for the direct 
sound could deteriorate ${ }^{1}$. For a specific acoustic environment, limiting the array radiation in the directions of the strongest reflections into the dark zone offers a more focused approach. Optimizing the array directivity so that the nulls are aligned with such directions is therefore a valid alternative to regularization. The nulls can be steered by adjusting source weights and positions with respect to the dark zone and surfaces ${ }^{22}$. This can be complementary to using directive sources.

By considering a reflection in the source weight optimization process, the ACC method will attenuate or cancel it at the control points in the dark zone. This process is subject to the same limitations as local dereverberation in room equalization (canceling or reducing any reflected signals at listening positions) ${ }^{23}$ or active noise control ${ }^{24}$. A common problem is a rapid drop of performance with increasing distance from the control points ${ }^{25,26}$. This can be alleviated by controlling both pressure and pressure gradient ${ }^{27,28}$, adding sensors ${ }^{25,29}$, preconditioning room responses (smoothing) ${ }^{30}$, or geometrical optimization to improve the match between the direct and reflected wavefronts at the control points ${ }^{31}$. The last method offers the most practical benefits, as it may improve attenuation away from the control locations without additional equipment or signal processing.

The above discussion indicates that ACC with optimized source positions is a potentially effective strategy for maximizing contrast with small systems when strong individual reflections occur in a room. Source position optimization will be the focus of Secs. IV and $\mathrm{V}$, after the background to the problem has been presented.

\section{B. Sound zone system under analysis}

Fig. 1 shows the sound zone system under analysis. The elementary geometrical scenario of two-dimensional zones and reflections propagating in the same plane is considered. Bright zone A is controlled by a single setup sensor and evaluated using $N$ monitor sensors. The geometrical center of the monitor sensor array coincides with the setup sensor. Dark zone B is

defined similarly. By defining distinct setup and monitor locations, the effect of performance 
drop with distance from the setup sensors ${ }^{26}$ can be included in the analysis and simulations bias in performance predictions is reduced ${ }^{32}$. There are two monopole sources whose spacing $d$ is small compared to the distances between the sources and any of the sensors. Each source is paired with an image generated by the reflecting rigid surface with a magnitude of reflection coefficient, $0 \leq \gamma \leq 1$. The distances between the source array center and the sensors in zone B are defined as $r_{B}$ and $s_{B n}$ for the setup and the monitor sensors respectively, where $n=1,2, \ldots, N$. The angles between the array axis and the lines between the source array center and the sensors in zone $\mathrm{B}$ are defined as $\phi_{B}$ and $\theta_{B n}$ for the setup and monitor sensors respectively. For the image sources, the distances and angles are defined by adding the superscript ' to the quantities defined for the physical array. Transfer functions between each source and the setup sensor in zone B, at a single frequency, are

$$
\begin{aligned}
& G_{B 1}=K\left(\frac{e^{-j k\left(r_{B}+d \cos \phi_{B} / 2\right)}}{r_{B}}+\frac{\gamma e^{-j k\left(r_{B}^{\prime}+d \cos \phi_{B}^{\prime} / 2\right)}}{r_{B}^{\prime}}\right), \\
& G_{B 2}=K\left(\frac{e^{-j k\left(r_{B}-d \cos \phi_{B} / 2\right)}}{r_{B}}+\frac{\gamma e^{-j k\left(r_{B}^{\prime}-d \cos \phi_{B}^{\prime} / 2\right)}}{r_{B}^{\prime}}\right),
\end{aligned}
$$

where $K=j \rho c k / 4 \pi$ in which $\rho$ is the air density, $c$ is the speed of sound, and $k$ is the wavenumber proportional to frequency. By analogy, transfer functions between each source and the $n$th monitor sensor in zone $\mathrm{B}$ are

$$
\begin{aligned}
& \Omega_{B n 1}=K\left(\frac{e^{-j k\left(s_{B n}+d \cos \theta_{B n} / 2\right)}}{s_{B n}}+\frac{\gamma e^{-j k\left(s_{B n}^{\prime}+d \cos \theta_{B n}^{\prime} / 2\right)}}{s_{B n}^{\prime}}\right), \\
& \Omega_{B n 2}=K\left(\frac{e^{-j k\left(s_{B n}-d \cos \theta_{B n} / 2\right)}}{s_{B n}}+\frac{\gamma e^{-j k\left(s_{B n}^{\prime}-d \cos \theta_{B n}^{\prime} / 2\right)}}{s_{B n}^{\prime}}\right) .
\end{aligned}
$$

It is convenient to define the sound pressure at each sensor using the principle of superposition. The complex pressure at the setup sensor in zone B, at a single frequency, can therefore be written as $p_{B}=\mathbf{G}_{B} \mathbf{q}$, where $\mathbf{G}_{B}=\left[G_{B 1}, G_{B 2}\right]$ and $\mathbf{q}=\left[q_{1}, q_{2}\right]^{T}$ is the vector of complex source weights. Similarly, sound pressure at the $n$th monitor sensor in zone B is $o_{B n}=\Omega_{B n} \mathbf{q}$, where $\Omega_{B n}=\left[\Omega_{B n 1}, \Omega_{B n 2}\right]$. For zone A, the distances, angles, transfer functions, and pressures are defined similarly. 


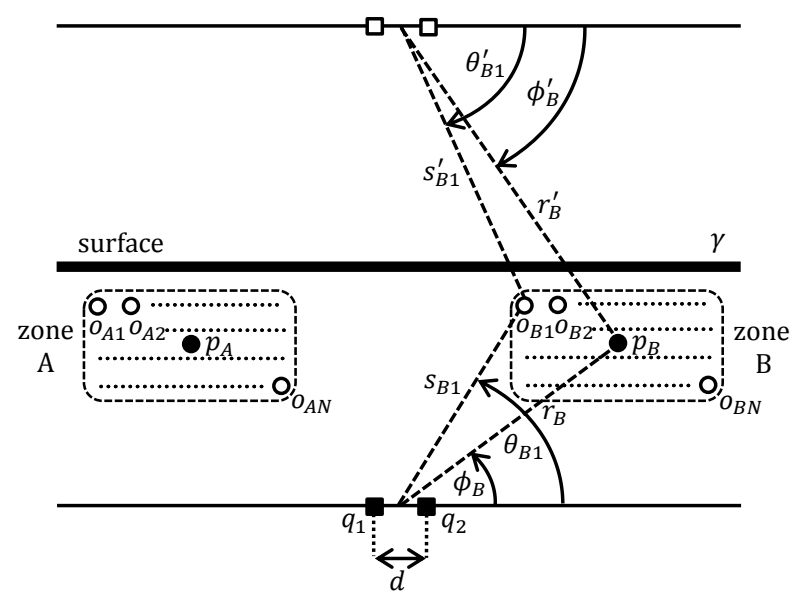

FIG. 1: A $2 \times 2$ sound zone system. Symbols: $\mathbf{a}$ source, $\square$ image source, - array axis, • setup sensor, o monitor sensor, w surface.

\section{Acoustic contrast}

The system's success can be quantified by evaluating the ratio of the sum of modulus squared sound pressures at monitor sensors in zone $\mathrm{A}$ and zone B. This ratio, known as acoustic contrast, is defined by

$$
\text { Contrast }=10 \log _{10}\left(\frac{\sum_{n=1}^{N}\left|o_{A n}\right|^{2}}{\sum_{n=1}^{N}\left|o_{B n}\right|^{2}}\right) .
$$

Large acoustic contrast indicates that the interference from the sound program targeted at zone $\mathrm{A}$ is acceptable for the listener in zone B (see Sec. I).

\section{Acoustic contrast control}

ACC is a sound energy control method that aims to maximize the acoustic contrast between the setup locations in zones $\mathrm{A}$ and $\mathrm{B}^{15}$. The optimal source weights can be found by solving a constrained optimization problem using the method of Lagrange multipliers. The squared pressure in zone B is to be minimized with the constraints that the squared

pressure in zone A and the sum of squared source weights (array effort) are set to certain 
chosen values. This leads to the following Lagrangian function:

$$
J=\mathbf{q}^{H} \mathbf{G}_{B}^{H} \mathbf{G}_{B} \mathbf{q}+\mu\left(\mathbf{q}^{H} \mathbf{G}_{A}^{H} \mathbf{G}_{A} \mathbf{q}-A\right)+\beta\left(\mathbf{q}^{H} \mathbf{q}-E\right),
$$

where $\mu$ and $\beta$ are the Lagrangian multipliers, and $A$ and $E$ are the chosen values of the squared pressure at the setup sensor in zone A and the array effort respectively. The minimum of function $J$ can be found by calculating partial differentials with respect to $\mathbf{q}, \mu$ and $\beta$ and setting them to zero, which yields

$$
\left(\mathbf{G}_{A}^{H} \mathbf{G}_{A}\right)^{-1}\left(\mathbf{G}_{B}^{H} \mathbf{G}_{B}+\beta \mathbf{I}\right) \mathbf{q}=\mu \mathbf{q}
$$

as well as $\mathbf{q}^{H} \mathbf{G}_{A}^{H} \mathbf{G}_{A} \mathbf{q}=A$ and $\mathbf{q}^{H} \mathbf{q}=E$. The optimal source weight vector is therefore proportional to the eigenvector $\hat{\mathbf{q}}$ corresponding to the largest eigenvalue of the matrix $\left(\mathbf{G}_{B}^{H} \mathbf{G}_{B}+\beta \mathbf{I}\right)^{-1}\left(\mathbf{G}_{A}^{H} \mathbf{G}_{A}\right)$ (after Elliott et $a l .{ }^{13}$ ). The constraint that the squared sound pressure in zone $\mathrm{A}$ must be equal to a value $A$ can be enforced by multiplying $\hat{\mathbf{q}}$ by an appropriate scaling factor and the effort constraint can be satisfied by adjusting the value of $\beta$. The latter therefore regularizes the matrix $\mathbf{G}_{B}^{H} \mathbf{G}_{B}$. Alternatively, $\beta$ can be set to a frequency independent value that is large enough to ensure validity of the numerical solution, for instance when the number of setup sensors in zone B is lower than the number of sources (in such cases, the matrix $\mathbf{G}_{B}^{H} \mathbf{G}_{B}$ is singular). For a $2 \times 2$ system, the regularized ACC solution is equivalent to crosstalk cancellation ${ }^{7}$ and $\mathrm{PM}^{11}$.

\section{Analytical Solution}

This section presents the analytical solution to the ACC problem. Hence, the expression is derived for acoustic contrast produced by the examined system.

\section{A. Optimal source weights}

The ACC problem can be solved analytically using the procedure outlined in Sec. II.D. For a general $2 \times 2$ system, a regularized solution must be derived ( $\mathbf{G}_{B}^{H} \mathbf{G}_{B}$ is singular). Eq. (5) was therefore used to find the elements of the unscaled optimal source weight vector $\hat{\mathbf{q}}$. 
The ratio of these elements formed an expression that included terms in the regularization parameter $\beta$. These terms were neglected assuming $\beta \rightarrow 0$. The resulting expression for the unit vector is $\hat{\mathbf{q}}=\frac{1}{Q}\left[G_{B 2},-G_{B 1}\right]^{T}$, where $Q=\sqrt{G_{B 2} G_{B 2}^{*}+G_{B 1} G_{B 1}^{*}}$ with the superscript * denoting a complex conjugate. Substituting this result into $\hat{p}_{B}=\mathbf{G}_{B} \hat{\mathbf{q}}$ shows that, with no regularization, the sources simply cancel the sound pressure at the setup sensor in zone B. ACC can therefore be considered as comprising direct and reflected sound cancelers.

\section{B. Acoustic contrast expression}

The complex sound pressure produced by the cancelers at the $n$th monitor sensor in zone B can be defined as $\hat{o}_{B n}=\Omega_{B n} \hat{\mathbf{q}}=\frac{1}{Q}\left(\Omega_{B n 1} G_{B 2}-\Omega_{B n 2} G_{B 1}\right)$. The squared sound pressure is therefore

$$
\begin{aligned}
\left|\hat{o}_{B n}\right|^{2} & =\hat{o}_{B n} \hat{o}_{B n}^{*}=\frac{1}{|Q|^{2}}\left(\left|\Omega_{B n 1}\right|^{2}\left|G_{B 2}\right|^{2}+\left|\Omega_{B n 2}\right|^{2}\left|G_{B 1}\right|^{2}\right. \\
& \left.-\Omega_{B n 1}^{*} \Omega_{B n 2} G_{B 1} G_{B 2}^{*}-\Omega_{B n 1} \Omega_{B n 2}^{*} G_{B 1}^{*} G_{B 2}\right)
\end{aligned}
$$

Substituting Eqs. (1) and (2) into Eq. (6) yields

$$
\begin{aligned}
\left|\hat{o}_{B n}\right|^{2} & =\frac{4|K|^{4}}{|Q|^{2}}\left[\left(\hat{O}_{B n}^{D}\right)^{2}+\left(\hat{O}_{B n}^{D^{\prime}}\right)^{2}+\left(\hat{O}_{B n}^{R}\right)^{2}+\left(\hat{O}_{B n}^{R^{\prime}}\right)^{2}\right. \\
& \left.+\hat{O}_{B n}^{D D^{\prime}}+\hat{O}_{B n}^{D R}+\hat{O}_{B n}^{D R^{\prime}}+\hat{O}_{B n}^{D^{\prime} R}+\hat{O}_{B n}^{D^{\prime} R^{\prime}}+\hat{O}_{B n}^{R R^{\prime}}\right],
\end{aligned}
$$

where $\hat{O}_{B n}^{D}, \hat{O}_{B n}^{D^{\prime}}, \hat{O}_{B n}^{R}, \hat{O}_{B n}^{R^{\prime}}, \hat{O}_{B n}^{D D^{\prime}}, \hat{O}_{B n}^{D R}, \hat{O}_{B n}^{D R^{\prime}}, \hat{O}_{B n}^{D^{\prime} R}, \hat{O}_{B n}^{D^{\prime} R^{\prime}}$, and $\hat{O}_{B n}^{R R^{\prime}}$ are the squared sound pressure components that arise due to the operation of the direct and reflected sound cancelers. The components are detailed in Table II. The superscripts $D$ and $R$ denote the relationship of a direct pressure component with the direct or reflected sound canceler respectively, and the superscripts $D^{\prime}$ and $R^{\prime}$ relate reflected pressure components with the

cancelers. The key components are: $\hat{O}_{B n}^{D}$ and $\hat{O}_{B n}^{D^{\prime}}$ - direct and reflected components due to direct sound canceler respectively; $\hat{O}_{B n}^{R}$ and $\hat{O}_{B n}^{R^{\prime}}$ - direct and reflected components due to reflected sound canceler respectively. It is noteworthy that these components can be related to directivity patterns of first-order differential arrays ${ }^{33}$. The remaining components depend on the key components. Eq. (7) and a similarly-defined squared sound pressure in zone A 
can be substituted to Eq. (3) to find contrast. Note that $4|K|^{2} /|Q|^{2}$ does not affect contrast, as it is a common factor in the resulting expression.

\section{Geometrical Optimization: Analysis}

In the following, the acoustic contrast expression is used to search geometrically for system configurations that maximize the contrast. The solution is dominated by the poles of Eq. (3) when sound pressure in zone B is close to zero. Eq. (7) is therefore analyzed to find source positions that minimize pressure in that zone. System geometries that increase the contribution of sound pressure in zone A to contrast are also investigated.

\section{A. Minimization of sound pressure in the dark zone}

The focus of this section is three techniques to minimize the squared sound pressure components that arise from analysis of the reflection in zone $\mathrm{B}$ and the operation of the reflection canceler: $\hat{O}_{B n}^{D^{\prime}}, \hat{O}_{B n}^{R}, \hat{O}_{B n}^{R^{\prime}}, \hat{O}_{B n}^{D D^{\prime}}, \hat{O}_{B n}^{D R}, \hat{O}_{B n}^{D R^{\prime}}, \hat{O}_{B n}^{D^{\prime} R^{\prime}}$, and $\hat{O}_{B n}^{R R^{\prime}}$. Component $O_{B n}^{D}$ produced by the direct sound canceler is also analyzed to inform the design of the systems' secondary degree of freedom.

1. Null-Split technique. Component $\hat{O}_{B n}^{D^{\prime}}$ can be reduced directly by appropriate orientation of the sources with respect to zone B. The orientation must be such that the difference $\cos \theta_{B n}^{\prime}-\cos \phi_{B}$, contained in $\hat{O}_{B n}^{D^{\prime}}$, is minimized. Fig. 2a shows an example configuration to achieve this aim. Ensuring that $\pm \phi_{B}=\mp \phi_{B}^{\prime}$, where $\phi_{B} \neq 0^{\circ}$, splits the directivity null generated by the physical array between the direct and reflected paths to the setup sensor in zone B; hence, the technique is referred to as the Null-Split. The principle is similar to null-splitting between two dark zones, discussed by Jones and Elliott ${ }^{5}$. When the condition $\pm \phi_{B}=\mp \phi_{B}^{\prime}$ is fulfilled, the value of $\cos \phi_{B}$ falls within the range of values of $\cos \theta_{B n}^{\prime}$ that is small for large distances of the image array from the zone. This is shown in Fig. 3a (top) for the configuration from Fig. 2a. The values of the cosine difference are therefore relatively 
(a) Null-Split: (b) Far-Align: (c) Near-Align:

$$
\begin{aligned}
\pm \phi_{B} & =\mp \phi_{B}^{\prime} & \phi_{B}=\phi_{B}^{\prime} & \phi_{B} \in\left\{0^{\circ}, 180^{\circ}\right\} \\
\phi_{B} & \neq 0^{\circ} & \phi_{B}^{\prime} & =\phi_{B}-180^{\circ}
\end{aligned}
$$

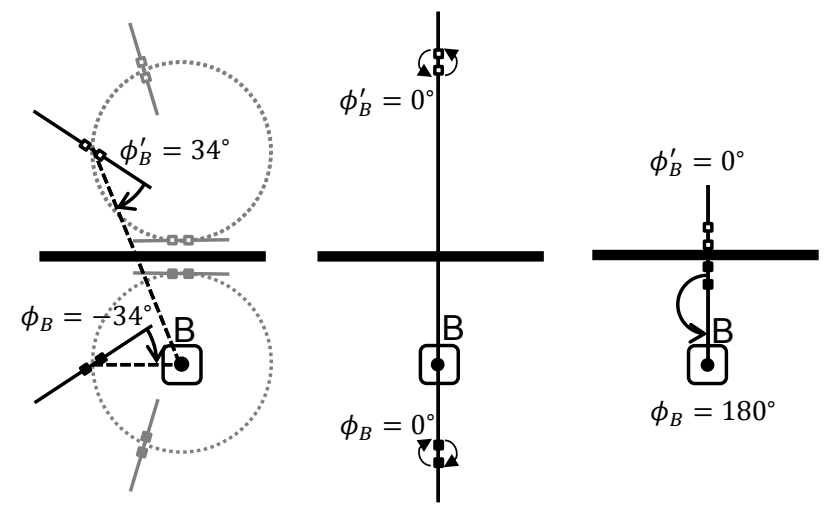

FIG. 2: Source positions for minimizing the reflected sound pressure in zone B. Symbols as in Fig. 1.

low: $-0.057 \leq\left(\cos \theta_{B n}^{\prime}-\cos \phi_{B}\right) \leq 0.053$. The condition $\pm \phi_{B}=\mp \phi_{B}^{\prime}$ deactivates the reflection canceler. The components $\hat{O}_{B n}^{R}, \hat{O}_{B n}^{R^{\prime}}, \hat{O}_{B n}^{D R}, \hat{O}_{B n}^{D R^{\prime}}, \hat{O}_{B n}^{D^{\prime} R^{\prime}}$, and $\hat{O}_{B n}^{R R^{\prime}}$ are therefore not produced. The only remaining reflection-related component is $\hat{O}_{B n}^{D D^{\prime}}$ which contains $\hat{O}_{B n}^{D^{\prime}}$ as a factor; hence, low values of $\hat{O}_{B n}^{D^{\prime}}$ contribute to the reduction of $\hat{O}_{B n}^{D D^{\prime}}$.

2. Far-Align technique. Components $\hat{O}_{B n}^{D^{\prime}}$ and $\hat{O}_{B n}^{D D^{\prime}}$ can also be reduced by aligning the sources with the setup sensor in zone B and the surface, as shown in Fig. 2b. The array is located further from the surface than the zone; therefore, the technique is referred to as the Far-Align. When the condition $\phi_{B}=\phi_{B}^{\prime}$ is met, the values of $\cos \phi_{B}$ are included in the range of the values of $\cos \theta_{B n}^{\prime}$, as shown in Fig. 3b (top) for the configuration from Fig. $2 \mathrm{~b}$. In this case, the endfire array significantly limits the range of $\cos \theta_{B n}^{\prime}$, leading to $-0.002 \leq\left(\cos \theta_{B n}^{\prime}-\cos \phi_{B}\right) \leq 0$; hence, larger reductions of the cosine difference values are achieved compared to the Null-Split and the attenuation of reflection is effective over a larger region. Non-endfire orientations of Far-Align are also possible, although they will attenuate over a smaller area. The condition $\phi_{B}=\phi_{B}^{\prime}$ deactivates the reflection canceler, similarly to 
(a) Null-Split

(b) Far-Align (c) Near-Align
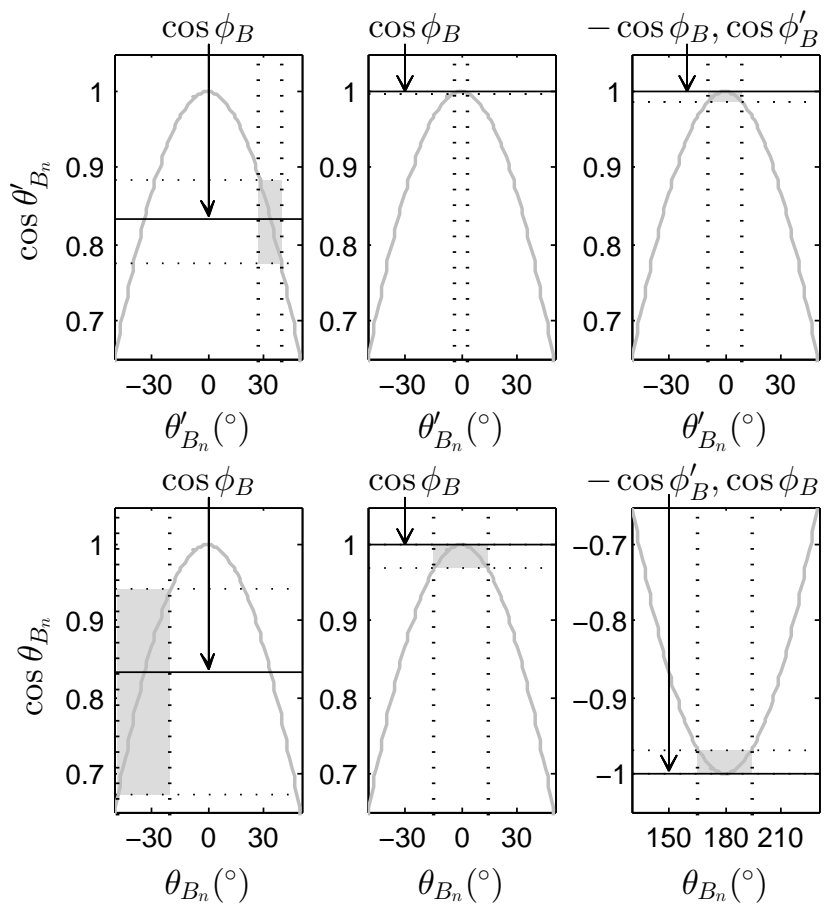

FIG. 3: Ranges of angles between the array axis and the monitor sensors in zone B, and the related cosine function values for configurations from Fig. 2 (indicated by the filled regions).

the Null-Split case.

3. Near-Align technique. Another way to reduce the reflection's impact in zone B is to use the reflection canceler. Its operation can be optimized by aligning the sources with the zone and surface, while ensuring that $\phi_{B} \in\left\{0^{\circ}, 180^{\circ}\right\}$ and $\phi_{B}^{\prime}=\phi_{B}-180^{\circ}$, as shown in Fig. 2c. Since the sources are located nearer to the surface than the zone, the method is referred to as the Near-Align. The alignment, the endfire orientation, and the large distance of the physical and image arrays from the zone result in $\cos \theta_{B n}^{\prime}-\cos \phi_{B} \approx-\left(\cos \theta_{B n}-\right.$ $\left.\cos \phi_{B}^{\prime}\right)$. This is demonstrated in Fig. 3c for the configuration from Fig. 2c. In this case, $1.986 \leq\left(\cos \theta_{B n}^{\prime}-\cos \phi_{B}\right) \leq 2$ and $-2 \leq\left(\cos \theta_{B n}-\cos \phi_{B}^{\prime}\right) \leq-1.970$ is observed. The large distance of the sources and images from the zone also results in $r_{B} \approx s_{B n}$ and $r_{B}^{\prime} \approx s_{B n}^{\prime}$, 
which in combination with the cosine relationship yields $\hat{O}_{B n}^{D^{\prime}} \approx-\hat{O}_{B n}^{R}$. This approximation is fundamental to active attenuation of the reflection, since it gives $\left(\hat{O}_{B n}^{D^{\prime}}\right)^{2}+\left(\hat{O}_{B n}^{R}\right)^{2}+\hat{O}_{B n}^{D^{\prime} R}$ $\approx 0$, as well as $\hat{O}_{B n}^{D D^{\prime}}+\hat{O}_{B n}^{D R} \approx 0$ and $\hat{O}_{B n}^{D^{\prime} R^{\prime}}+\hat{O}_{B n}^{R R^{\prime}} \approx 0$, which according to Eq. (7) reduces the influence of $\hat{O}_{B n}^{D^{\prime}}$ and $\hat{O}_{B n}^{R}$, and the associated components in zone B. The Near-Align also yields $\cos \theta_{B n}^{\prime}-\cos \phi_{B}^{\prime} \approx 0$, which reduces the remaining reflection-related components $\hat{O}_{B n}^{R^{\prime}}$ and $\hat{O}_{B n}^{D R^{\prime}}$. The configuration from Fig. $2 \mathrm{c}$ achieves $-0.014 \leq\left(\cos \theta_{B n}^{\prime}-\cos \phi_{B}^{\prime}\right) \leq 0$, as shown in Fig. 3c (top).

The properties of Near-Align indicate that an extended attenuation region is produced. This characteristic was previously demonstrated by Guo and $\mathrm{Pan}^{31}$ for an active noise control system based on a pair of widely spaced sources aligned with a single sensor, and forming the right angle with the reflecting surface. The zone broadening was attributed to the match between the direct and reflected wavefronts around the sensor. The Near-Align technique extends the wavefront matching principle to a compact source arrangement. Moreover, the impact of the principle on the bright zone sound field and the acoustic contrast has not been examined - these problems is addressed in Secs. V.B and V.C.

4. Attenuation of direct sound. Component $\hat{O}_{B n}^{D}$ can be reduced using a source array that is endfire with respect to the setup sensor in zone B, and located at a large distance. This results in $\cos \theta_{B n}-\cos \phi_{B} \approx 0$ and therefore small values of $\hat{O}_{B n}^{D}$, as demonstrated in Figs. 3b and 3c (bottom) for the configurations from Figs. 2b and 2c respectively. Far-Align results in $-0.030 \leq\left(\cos \theta_{B n}-\cos \phi_{B}\right) \leq 0$, and Near-Align gives $0 \leq\left(\cos \theta_{B n}-\cos \phi_{B}\right) \leq 0.030$. In contrast, the Null-Split array yields $-0.159 \leq\left(\cos \theta_{B n}-\cos \phi_{B}\right) \leq 0.110$, as shown in Fig. 3a (bottom). A smaller range of values for Far- and Near-Align indicates broadening of the direct sound attenuation area. Elliott and Jones ${ }^{4}$ demonstrated such capability in simulations for a pair of closely-spaced, free-field monopoles with endfire orientation in a similar system. 


\section{B. Bright zone considerations}

While attenuating sound in zone B is of primary importance for producing large contrast, the influence of the above techniques on the sound pressure in zone A must also be examined. Source weights $\hat{\mathbf{q}}$ will result in a low efficiency of radiation into zone A at low frequencies, which increases the array effort of the scaled source weights $\mathbf{q}^{13,16}$. To minimize the effort and achieve large contrast, the produced SPL peaks should coincide with zone $\mathrm{A}^{5}$. With a single surface, the peaks maintain a high DRR, and so collocating a maximum of the direct sound radiation with zone $\mathrm{A}$ is a valid means of enhancing contrast and reducing effort. Jones and Elliott ${ }^{5}$ observed that for a pair of free-field monopoles with the ACC weights, the position of directivity peaks changed with the array orientation with respect to the dark zone. Although these results indicate suitable zone A locations for the examined system, it is desirable to define precise geometrical requirements that must be met to increase this zone's pressure contribution to contrast.

When determining the required position for zone A, it is sensible to consider the geometrical center of the monitor sensor array, which coincides with the setup sensor. The direct component of the unscaled squared pressure at the setup sensor in zone $\mathrm{A},\left(\hat{O}_{A}^{D}\right)^{2}$, is defined similarly to the component $\left(\hat{O}_{B n}^{D}\right)^{2}$ from Table II. Fig. 4a shows $\left(\hat{O}_{A}^{D}\right)^{2}$, normalized and plotted in decibels against $\cos \theta_{A}-\cos \phi_{B}$ and $k d$, that characterize the component's dependence on the angular distance from zone B and on frequency respectively. The range of $\cos \theta_{A}-\cos \phi_{B}$ covers all angular distances, and $k d$ extends up to the spatial aliasing limit of the source array. The pressure decreases by no more than $6 \mathrm{~dB}$ with respect to the maximum in at least $2 / 3$ of the frequency range for $\cos \theta_{A}-\cos \phi_{B} \leq-1$ or $\cos \theta_{A}-\cos \phi_{B} \geq 1$ (outside of the grayed-out region of Fig. $4 \mathrm{a})$. For $-180^{\circ} \leq \phi_{B} \leq-90^{\circ}$ or $90^{\circ} \leq \phi_{B} \leq 180^{\circ}$, the inequalities hold if $-\arccos \left(1+\cos \phi_{B}\right) \leq \theta_{A} \leq \arccos \left(1+\cos \phi_{B}\right)$, whereas for $-90^{\circ} \leq \phi_{B} \leq 90^{\circ}$ we must have $-180^{\circ} \leq \theta_{A} \leq-\arccos \left(-1+\cos \phi_{B}\right)$ or $\arccos \left(-1+\cos \phi_{B}\right) \leq \theta_{A} \leq 180^{\circ}$. Such ranges of $\theta_{A}$ are indicated by the white regions in Fig. $4 \mathrm{~b}$ (results that will be discussed in Sec. V are also included). Large pressures in zone A are therefore achieved if the zone's center is 
(a)

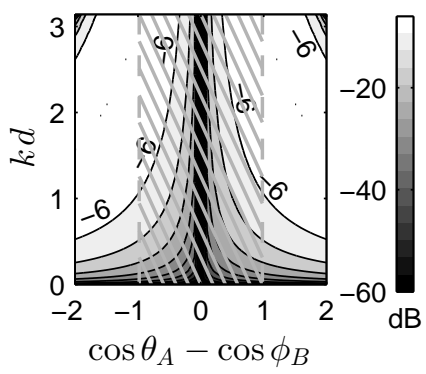

(b)

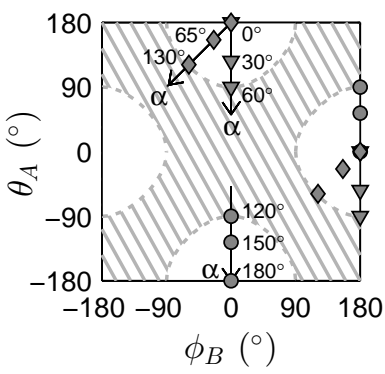

FIG. 4: (a) The normalized direct squared sound pressure component at the setup sensor in zone $\mathrm{A},\left(\hat{O}_{A}^{D}\right)^{2}$ (decibel scale with a $-60 \mathrm{~dB}$ limit). Outside the grayed-out region, $10 \log _{10}\left(\left(\hat{O}_{A}^{D}\right)^{2}\right) \geq-6 \mathrm{~dB}$ in at least $2 / 3$ of the frequency range. (b) Ranges of $\theta_{A}$ that increase the contribution of pressure in zone A to contrast (white regions). Arrays corresponding to the marked values in Fig. 6: Null-Split, $\boldsymbol{\nabla}$ Far-Align, $\bullet$ Near-Align.

located on the other side of the axis normal than zone B's setup sensor, and within the required range of $\theta_{A}$ that is the largest for $\phi_{B} \in\left\{0^{\circ}, \pm 180^{\circ}\right\}$ and decreases as $\phi_{B} \rightarrow \pm 90^{\circ}$; in the limit, the most suitable locations for zone A center are perpendicular to the axis normal (in either direction). Note that except for this special case, for any given value of $\phi_{B}$ the required range of $\theta_{A}$ consists of pairs of identical values with opposite signs. This means that zone A center can be suitably located on either side of the array axis.

\section{Geometrical Optimization: Simulations}

Having derived analytic techniques for maximizing the acoustic contrast, simulations are now presented. First, the Null-Split, Far-Align, and Near-Align techniques are compared for a $2 \times 2$ system in various configurations. The results are used to formulate guidelines for choosing the most suitable technique for a given position and orientation of the surface with respect to the zones. Second, numerical optimization is employed to validate the techniques and test their applicability to systems with additional control sensors, sources, and surfaces. 
Finally, the optimized systems are evaluated for contrast and robustness to implementation errors.

\section{A. Null-Split, Far-Align, and Near-Align comparison}

Fig. 5 shows the $2 \times 2$ system with a surface in various positions and orientations, covering the full range of possible scenarios. The surfaces were defined as lines tangent to a semi-circle with the radius of $4.25 \mathrm{~m}$ and centered at $(0.75 \mathrm{~m},-1.25 \mathrm{~m})$, which were spaced by $5^{\circ}$. In each case, the frequency independent magnitude of the reflection coefficient was set to $\gamma=1$, which provided a worst-case perspective on the effects of reflections. The two square zones, $\mathrm{A}$ and $\mathrm{B}$, each contained one setup and thirty-six monitor sensors with $5 \mathrm{~cm}$ spacing. For each surface, Null-Split, Far-Align, and Near-Align were evaluated. An array of two sources with $d=5 \mathrm{~cm}$ spacing was moved around a circle of $2 \mathrm{~m}$ radius, centered at the zone B's setup sensor, to form the Far- and Near-Align arrangements. The Null-Split was produced by rotating the array located halfway between the zones. In this location, the requirements for a large contribution of zone A's pressure to contrast, defined in Sec. IV.B, were satisfied for a large number of surface positions.

Source weights were determined using regularized ACC (Sec. II.D). The regularization was frequency independent with $\beta=10^{-6}$, which was the smallest value that avoided singularity in the numerical solutions. This type of regularization parallels the approach from the analysis in Secs. III and IV, where $\beta$ was assumed to be negligible or infinitesimal. The squared pressures in each zone were calculated for forty-four frequency bins with one-twelfth octave band spacing in the range $250-3175 \mathrm{~Hz}$. The motivation behind the logarithmic spacing was to avoid bias from regular variations of pressure over frequency. To evaluate the system, the frequency-averaged squared pressure was calculated at each monitor sensor. When calculating the averages, values at each frequency were linearly weighted to compensate for the logarithmic spacing. The contrast was then obtained using Eq. (3).

Fig. 6 shows the contrast plotted against surface position. The marked values, contained 


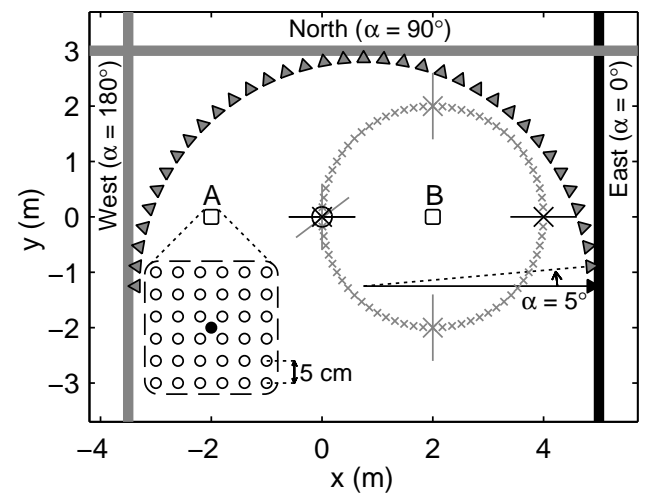

FIG. 5: A $2 \times 2$ system with a surface in a number of locations. The surface is tangent to the semi-circle at each angular position indicated by the arrowhead $\boldsymbol{\Lambda}$. Remaining symbols: zone, $\times$ source array center: Far- and Near-Align, $\otimes$ source array center: Null-Split, — example array axis, example surface; in close-up: • setup sensor, o monitor sensor.

in and bounding the thick portions of the contrast curves, were produced by the arrays enclosed in the white regions in Fig. 4b (note that each array's location can be determined by the angles interpreted in either direction from the array axis). Therefore, the thick portions of the curves correspond to the arrays that meet the positioning requirements for the increased contribution to contrast of sound pressure in zone A. These arrays achieve high contrasts (over $28 \mathrm{~dB}$ ). Each technique has a range where it performs better than the others: Far-Align in $0^{\circ} \leq \alpha \leq 80^{\circ}$, Null-Split in $85^{\circ} \leq \alpha \leq 120^{\circ}$, and Near-Align in $125^{\circ} \leq \alpha \leq 180^{\circ}$. Considering the surface positions for which the techniques lead, the following design guidelines can be formulated: (i) if the distance of both zones from the surface is the same or similar, the sources should be positioned according to the Null-Split rule; (ii) if the surface is closer to zone B than zone A, the Far-Align arrangement should be chosen; and (iii) if zone $\mathrm{A}$ is closer to the surface than zone B, the Near-Align technique applies.

For surfaces in ambiguous positions, a closer examination of geometrical options should identify the best technique. In such cases, the arrays that fulfill the positioning requirements for zone A should be the first choice, and the Far- and Near-Align should be preferred over 


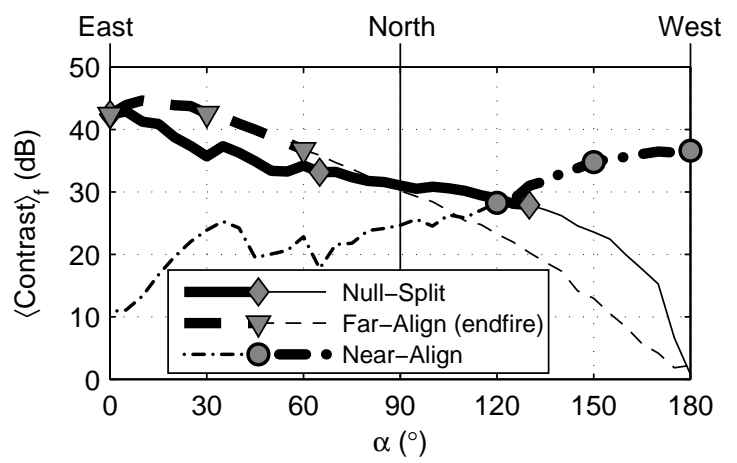

FIG. 6: Frequency averaged acoustic contrast produced by the Null-Split, Far-Align, and Near-Align arrays with a surface in different positions, as shown in Fig. 5. The arrays producing the marked contrast values are indicated in Fig. 4b, thus the thick portions of the curves correspond to arrangements that increase the contribution of pressure in zone A to contrast.

Null-Split. According to Fig. 6, combining these selection criteria with the general guidelines selects the best performing arrays for all the surfaces, except for $65^{\circ} \leq \alpha \leq 80^{\circ}$ where zone $\mathrm{A}$ is outside the required range for Far-Align, yet this solution outperforms the Null-Split. This can be attributed to less effective attenuation in zone B by non-endfire Null-Split arrays, as discussed in Sec. IV.A. However, the contrast differences between the two techniques in this range are relatively small (less than $3 \mathrm{~dB}$ ).

\section{B. Numerical optimization of source positions}

In this section, source positions are optimized by numerical search. This method has advantages: (i) it provides independent validation of the proposed techniques; (ii) it has capacity to reveal alternative techniques potentially overlooked in the analysis; (iii) it allows straightforward extensions (additional sensors, sources, and surfaces); and (iv) it is directly applicable to practical implementations. The considered systems are $2 \times 2,2 \times 50$, and $3 \times 50$, with one or two surfaces. Fig. 7 shows the geometries considered in the optimization process. There were three surfaces, each with $\gamma=1$, considered either individually or in combination. 


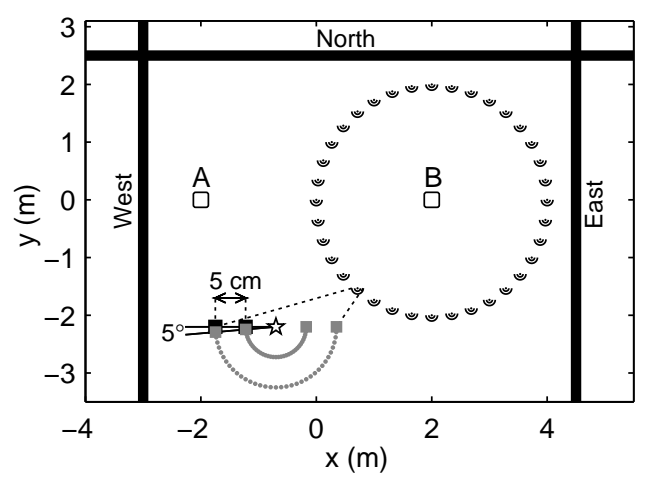

FIG. 7: Configuration used in the numerical search for optimal source positions. Symbols: zone, U source subset, $\mathbf{m}$ surface; in close-up: is fixed source, $\mathbf{a}$ candidate source.

For the $2 \times 2$ system, the same sensor layouts as in Fig. 5 were used. For the $2 \times 50$ and $3 \times 50$ systems, there were twenty-five setup sensors in each zone, arranged on square $5 \mathrm{~cm}$ grids that were centered at the setup sensor positions used for the $2 \times 2$ system. There were thirty-six subsets of candidate sources located around zone B on a $2 \mathrm{~m}$ radius circle $\left(10^{\circ}\right.$ interval). Each subset contained a fixed source and candidate sources located on the inner and outer arcs around that source. There were thirty-seven candidate sources on each arc ( $5^{\circ}$ separation). In the $2 \times 2$ and $2 \times 50$ cases only the inner arc was considered, whereas for the $3 \times 50$ system the candidates from both arcs were used.

The optimization procedure was based on the principle of a beam search: a small number of solutions were developed in parallel to increase the probability of finding a good solution with minimal search effort ${ }^{34}$. First, each subset was tested for the best array orientation. For the $2 \times 2$ and $2 \times 50$ systems, this meant choosing the best out of thirty-seven source pairs formed by the fixed source and each of the candidate sources located on the inner arc. In the $3 \times 50$ case, the optimization algorithm chose the best of thirty-seven triples formed by the fixed source and aligned pairs of candidates located on the two arcs. The overall best performing configuration was then selected from the thirty-six preselected pairs or triplets. The objective function was the frequency-averaged acoustic contrast, calculated as in Sec. V.A. The source weights were determined similarly as in Sec. V.A and scaled to 
(a) North: Null-Split

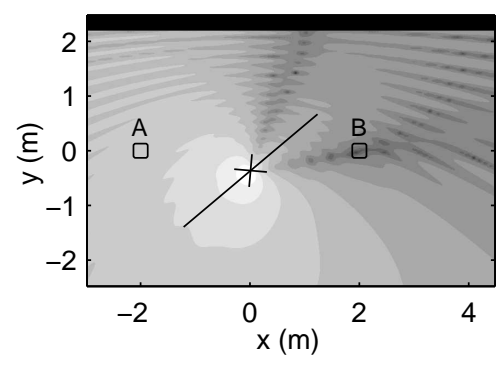

(b) East: Far-Align

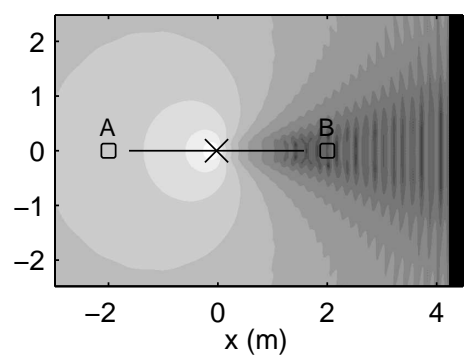

(c) West: Near-Align

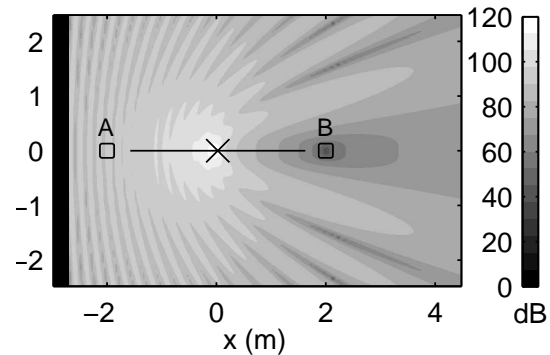

FIG. 8: Optimized source arrays for a $2 \times 2$ system with a single surface in different locations: (a) North, (b) East and (c) West, and the sound pressure level maps at $1 \mathrm{kHz}$. Symbols: $\square$ zone, $\times$ array center, - array axis, $\square$ surface.

produce $94 \mathrm{~dB}$ SPL in zone A (measured as the average SPL at the setup sensors).

1. $2 \times 2$ system with a single surface. Fig. 8 shows the optimization results for a $2 \times 2$ system with a surface in three different positions, overlaid on the SPL maps for the optimized arrays at $1 \mathrm{kHz}$. The Null-Split, Far-Align, and Near-Align arrangements were chosen as optimal for the North, East, and West surfaces respectively, which demonstrates the validity of these solutions. The Null-Split produces a narrower null in zone B than the Far- and Near-Align, which was indicated by the analytic results in Sec. IV.A. The array selection follows the general guidelines from Sec. V.A. It was verified that all three arrays fulfilled the positioning requirements for increased contribution of pressure in zone A to contrast, which confirms that it is an important factor in the selection.

2. $2 \times 50$ system with a single surface. For the East and West surfaces, the sources selected for the $2 \times 2$ and $2 \times 50$ systems were identical. This demonstrates the applicability of the analytic Far- and Near-Align solutions to systems with extended setup sensor arrays. A different solution was chosen in the North case, where the array approximated Far-Align, as shown in Fig. 9a. The sources were shifted from the regular Far-Align position towards 
(a) 2×50: Approx.

Far-Align

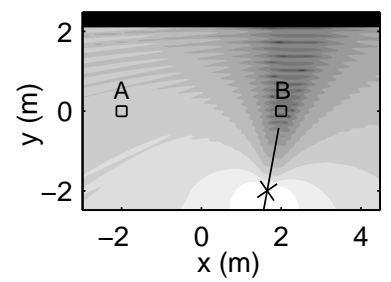

(b) $3 \times 50$ :

Near-Align

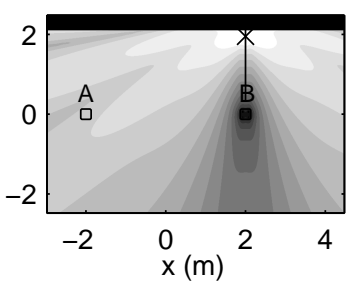

(c) $2 \times 2$ : Far-Align

and Null-Split

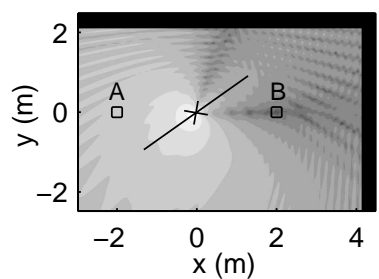

(d) $2 \times 2$ : Far-Align and Near-Align

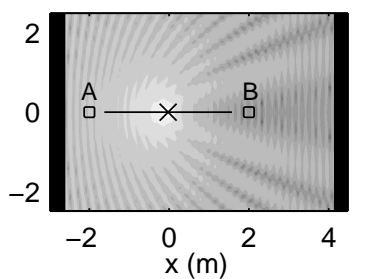

FIG. 9: Optimized source arrays with surfaces generating first order reflections, and the sound pressure level maps at $1 \mathrm{kHz}$ : (a) $2 \times 50$, North; (b) $3 \times 50$, North; (c) $2 \times 2$, East and North; and (d) 2×2, East and West surface locations. Symbols as in Fig. 8 .

zone A and rotated to remain endfire at zone B. Neither the Far-Align arrangement nor the adjusted array met the positioning requirements for zone A. However, the adjustment alleviated the effect of unwanted sound attenuation in that zone, increasing contrast at low frequencies. The preference of this solution over Null-Split from Fig. 8a can be attributed to null broadening, which improved contrast at middle and high frequencies.

3. $3 \times 50$ system with a single surface. For the East and West surfaces, the positions and orientations of the arrays chosen for the $3 \times 50$ system were the same as for $2 \times 2$ and $2 \times 50$, and in the North case the Near-Align arrangement was selected. These results indicate the suitability of the Far- and Near-Align techniques for larger source arrays. The North case is shown in Fig. 9b. The array does not satisfy the positioning requirements for zone A - the direct sound in that zone is excessively attenuated, but this is compensated by strong reflections produced by the array located close to the surface. These reflections interfere with the direct sound, creating pressure nulls in zone A periodically at certain frequencies. However, the influence of these nulls on contrast is balanced out by effective cancellation - Near-Align facilitates destructive interference between the direct and reflected sound, producing a localized minimum in zone B. 
4. Systems with two surfaces. Figs. $9 \mathrm{c}$ and $9 \mathrm{~d}$ show the optimization results for the $2 \times 2$ system with two surfaces generating first order reflections. Fig. 9c shows the case with two perpendicular surfaces. This layout combines the Far-Align (non-endfire) and the NullSplit solutions for the East and North surfaces respectively. In Fig. 9d, two surfaces are positioned in parallel. The chosen array forms the Far- and Near-Align arrangements for the East and West surfaces respectively. For $2 \times 50$ and $3 \times 50$ systems with the same surface combinations, the optimized source arrays had positions and orientations that were similar to the $2 \times 2$ case. These results demonstrate that combining the Null-Split, Far-Align and Near-Align techniques is a valid optimization approach when two strongly reflecting surfaces coexist.

\section{Evaluation of the optimized systems}

In this section, the optimal arrays from Sec. V.B are evaluated for contrast and compared with systems that represent different approaches to sound zone generation under reflective conditions. For each system, three stages can be distinguished in the process: source position optimization, source weights calculation, and sound field evaluation. At each stage, the acoustic conditions can be either anechoic (abbreviated to A) or reflective (R). The systems described in Sec. V.B are ACC-based, with reflective conditions at all stages (ACC Reflective-Reflective-Reflective). These are compared with fully anechoic (ACC Anechoic-Anechoic-Anechoic) and mixed conditions (ACC A-A-R and ACC A-R-R) systems. Furthermore, the performance of the arrays that actively attenuate reflections with non-optimized source positions is evaluated by calculating the median (M) of contrasts achieved by all the configurations considered in the optimizations (ACC M-R-R). Finally, the aforementioned SPM method with source positions optimized for maximum contrast is evaluated (SPM R-R-R).

The ACC A-A-A arrays have source positions and weights optimized for maximum contrast under anechoic conditions. With matching ideal conditions at the evaluation stage, 
they define the upper performance limit. Comparing the ACC M-R-R, ACC A-A-R, ACC A-R-R, ACC R-R-R, and SPM R-R-R results with this limit will rank a number of different approaches to sound zone generation under reflective conditions. The ACC M-R-R results will indicate the probable effect of attenuating reflections using an arbitrarily chosen configuration (there is equal chance that contrast will be higher or lower than the median value). Evaluating the ACC A-R-R and ACC A-A-R systems will show the effect of using sources optimized for the direct sound only under reflective conditions, either with (A-R-R) or without the reflection canceler (A-A-R). The performance of the ACC R-R-R systems will demonstrate the impact of combining the reflection canceler and sources optimized for the reflective conditions. Finally, the SPM R-R-R results will show the effectiveness of using the power minimization approach to reduce the impact of individual reflections on contrast. SPM adjusts the source weights, taking reflections into account, to minimize the sound power radiated by the array while aiming to maintain a high SPL in zone $\mathrm{A}^{5,16}$. Minimizing the power should reduce the strength of reflections in the dark zone, thus improving contrast. Further improvement should be obtained from optimizing the source positions for maximum contrast.

Table III shows the frequency-averaged contrast for the $2 \times 2,2 \times 50$, and $3 \times 50$ systems. In all cases, the ACC R-R-R systems achieved the highest contrasts (results in boldface), exhibiting the smallest degradation of performance with respect to ACC A-A-A. While an average $18.6 \mathrm{~dB}$ contrast loss was observed for ACC R-R-R configurations in the single surface scenario, in the ACC M-R-R, ACC A-A-R, ACC A-R-R, and SPM R-R-R cases the contrast was degraded on average by $58.9 \mathrm{~dB}, 42.7 \mathrm{~dB}, 33.9 \mathrm{~dB}$, and $52.9 \mathrm{~dB}$ respectively. This demonstrates the benefits of source optimization using the Null-Split, Far-Align, and Near-Align techniques and their approximations. Moreover, these results indicate that ACC implemented on a geometrically optimized array, even for direct sound only, is a more suitable sound zone reproduction strategy than SPM when strong individual reflections occur. Comparison of the ACC A-R-R and ACC R-R-R contrasts for the systems with two surfaces shows an average 9.6 dB gain when using configurations that combine Null-Split, 
Far-Align, and Near-Align instead of sources optimized considering the direct sound only.

\section{Robustness to implementation errors}

The proposed source optimization techniques will be subject to implementation errors in practice. This section investigates the techniques' sensitivity to source and surface positioning, and to reflectivity errors. The techniques are represented by $2 \times 2$ arrangements optimized for a single surface (ACC R-R-R) from Sec. V.C, and compared with SPM (SPM R-R-R). Random source and surface position errors were drawn from independent normal distributions for $x$ and $y$ coordinates. Normally-distributed errors to the surface reflection coefficients were introduced similarly. In each case, the procedure was repeated for 100 trials and the mean acoustic contrast over all trials was obtained.

The source position errors had one standard deviation (1 s.d.) of $0.25 \mathrm{~mm}$ and $5 \mathrm{~mm}$, which can be regarded as manufacturing tolerance versus manual placement. Surface errors had 1 s.d. of $1 \mathrm{~mm}$ and $100 \mathrm{~mm}$, which represent minor and major displacement in relative position. The reflection coefficient was perturbed from the default value $\gamma=0.8$ with 1 s.d. of 0.02 and 0.05 . Errors were applied before or after source weight calculation, i.e., calibrated errors at setup or uncalibrated error at playback.

It was found that the techniques are generally most sensitive to source position errors, although the contrast degrades gracefully as in anechoic conditions. For instance, for the calibrated Null-Split technique (ACC R-R-R, North), the largest source and surface errors resulted in contrasts of $25.2 \mathrm{~dB}$ and $32.4 \mathrm{~dB}$ (losses of $8.1 \mathrm{~dB}$ and $0.9 \mathrm{~dB}$ ) respectively. Calibrating the ACC techniques' source weights typically recovered at least $2 \mathrm{~dB}$ contrast with respect to uncalibrated position errors (improvement could also be achieved by appropriate regularization $^{1}$ ). This gain was most pronounced for Near-Align (ACC R-R-R, West): e.g., the largest source error gave $33.0 \mathrm{~dB}$ calibrated ( $2.3 \mathrm{~dB}$ loss) versus $17.0 \mathrm{~dB}$ uncalibrated (18.3 dB loss). The largest surface error produced $34.1 \mathrm{~dB}$ calibrated (1.2 dB loss) compared to $10.5 \mathrm{~dB}$ uncalibrated (24.8 dB loss), as the Near-Align technique relies 
on destructive interference between direct and reflected sound for cancellation in the dark zone. From its much lower ideal-case performance, SPM was generally less sensitive to errors in terms of contrast than the ACC techniques, which nevertheless outperformed SPM in all error conditions except for uncalibrated surface errors in the ACC R-R-R, West case. Overall, the ACC techniques gave twice the contrast of SPM with large position errors. The effect of reflection coefficient errors was negligible in all cases (less than $1 \mathrm{~dB}$ ). These results support the previous conclusion that ACC combined with the proposed source optimization techniques is the most effective sound zoning strategy with strong individual reflections for a limited number of sources.

\section{E. Discussion}

The findings from Secs. V.A-V.D may have significant implications for the design of sound zone systems. First, the Null-Split, Far-Align, and Near-Align techniques, selected according to the guidelines from Sec. V.A, are valid strategies for limiting the impact of strong reflections from a single surface on acoustic contrast. This was demonstrated for a $2 \times 2$ system in Sec. V.B.1. Second, the Far- and Near-Align arrangements, or their approximations, can maximize contrast for systems with a larger number of setup sensors and sources, as shown in Secs. V.B.2 and V.B.3. This result may influence the specification of candidate source locations when optimizing large systems, by indicating alternatives to conventional linear or circular arrangements. Third, the Null-Split, Far-Align, and NearAlign techniques can provide a framework for the optimization strategy in cases when strong reflections are generated by more than one surface, as shown in Sec. V.B.4. Finally, ACC combined with the Null-Split, Far-Align, and Near-Align techniques have the potential to realize substantial gains in contrast with respect to alternative approaches to sound zone generation when strong isolated reflections are present, as demonstrated by the results from Secs. V.C and V.D. Such a combination may therefore be utilized to avoid passive acoustic treatment on the problematic surfaces. 


\section{Summary}

Optimization of source positions for the ACC-based small sound zone systems with strong individual reflections was investigated. Analytical study of a $2 \times 2$ system resulted in three techniques that reduce the reflected sound pressure in the dark zone: Null-Split (array optimally oriented with respect to the dark zone and surface); Far-Align (array aligned away from the dark zone and surface); and Near-Align (array aligned between the dark zone and surface). Source locations that increase the contribution of the bright zone sound pressure to contrast were also determined analytically. The analytical findings provided the basis for a set of design guidelines for system configuration, developed using simulations: Null-Split should be used for zones at the same or similar distance to the surface, whereas the Farand Near-Align configurations are most suitable when the surface is closer to the dark or bright zone respectively.

Numerical optimizations validated the analytical solutions and demonstrated their applicability to larger systems and to the condition when two surfaces give strong reflections. The arrays optimized using the Null-Split, Far-Align, and Near-Align techniques, or their approximations, limited the contrast deterioration with respect to the upper limit achieved under anechoic conditions to $19 \mathrm{~dB}$ on average, outperforming the SPM method by $34 \mathrm{~dB}$. Future work could include experimental validation of the techniques and investigations on higher order source arrays and reflections. The influence of the techniques on the perception of sound in the zones should also be examined, for instance by investigating the acceptability or distraction of the interfering sound programs ${ }^{2,35}$.

\section{Acknowledgments}

This research was supported by Bang \& Olufsen A/S and EPSRC DTG as a part of the POSZ project. The authors thank all members of the project and Wenwu Wang from the University of Surrey for useful discussions. 


\section{References}

1 P. Coleman, P. J. B. Jackson, M. Olik, M. Møller, M. Olsen, and J. Pedersen, "Acoustic contrast, planarity and robustness of sound zone methods using a circular loudspeaker array", J. Acoust. Soc. Am. 135, 1929-1940 (2014).

2 J. Francombe, R. Mason, M. Dewhirst, and S. Bech, "Determining the threshold of acceptability for an interfering audio programme", in Proc. AES 132nd Conv., Budapest, 26-29 April 2012.

3 W. F. Druyvesteyn and J. Garas, "Personal sound", J. Audio Eng. Soc. 45, 685-701 (1997).

4 S. J. Elliott and M. Jones, "An active headrest for personal audio", J. Acoust. Soc. Am. 124, 2702-2709 (2006).

5 M. Jones and S. J. Elliott, "Personal audio with multiple dark zones", J. Acoust. Soc. Am. 124, 3497-3506 (2008).

6 J.-H. Chang, C.-H. Lee, J.-Y. Park, and Y.-H. Kim, "A realization of sound focused personal audio system using acoustic contrast control", J. Acoust. Soc. Am. 125, 20912097 (2009).

7 J.-Y. Park, J.-H. Chang, and Y.-H. Kim, "Generation of independent bright zones for two-channel private audio system", J. Audio Eng. Soc. 58, 382-393 (2010).

8 M. Shin, S. Q. Lee, F. M. Fazi, P. A. Nelson, D. Kim., S. Wang, K.-H. Park, and J. Seo, "Maximization of acoustic energy difference between two spaces", J. Acoust. Soc. Am. 128, 121-131 (2010).

9 F. Jacobsen, M. Olsen, M. Møller, and F. Agerkvist, "A comparison of two strategies for generating sound zones in a room", in Proc. 18th Int. Congress Sound Vib., Rio de Janeiro, 10-14 July 2011.

10 J. Cheer, "Active control of the acoustic environment in automobile cabin", Ph.D. thesis, chapts. 7-8, Univ. of Southampton (2012), retrieved from eprints.soton.ac.uk/348819/ (viewed 4/25/2014). 
11 M. F. Simón-Gálvez, S. J. Elliott, and J. Cheer, "A superdirective array of phase shift sources", J. Acoust. Soc. Am. 132, 746-756 (2012).

12 M. Olik, J. Francombe, P. Coleman, P. J. B. Jackson, M. Olsen, M. Møller, R. Mason, and S. Bech, "A comparative performance study of sound zoning methods in a reflective environment", in Proc. AES 52nd Int. Conf., Guildford, UK, 2-4 September 2013.

13 S. J. Elliott, J. Cheer, J.-W. Choi, and Y. Kim, "Robustness and regularization of personal audio systems", IEEE Trans. Audio Speech Lang. Proc. 20, 2123-2133 (2012).

14 Y. Wen, J. Yang, and W.-S. Gan, "Strategies for acoustical-hotspot generation", IEICE Trans. Fundamentals of Electronics, Communications and Computer Sciences E88, 1739$1746(2005)$.

15 J.-W. Choi and Y.-H. Kim, "Generation of an acoustically bright zone with an illuminated region using multiple sources", J. Acoust. Soc. Am. 111, 1695-1700 (2002).

16 S. J. Elliott, J. Cheer, H. Murfet, and K. R. Holland, "Minimally radiating sources for personal audio", J. Acoust. Soc. Am. 128, 1721-1728 (2010).

17 J.-H. Chang and F. Jacobsen, "Sound field control with a circular double-layer array of loudspeakers", J. Acoust. Soc. Am. 131, 4518-4525 (2012).

18 C. Díaz and A. Pedrero, "The reverberation time of furnished rooms in dwellings", Appl. Acoustics 66, 945-956 (2005).

19 R. Walker, "A controlled-reflection listening room for multi-channel sound", in Proc. 104th AES Conv., Amsterdam, 16-19 May 1998.

20 A. Sæbø, "Influence of reflections on crosstalk cancelled playback of binaural sound", Ph.D. thesis, chapt. 4, Norwegian Univ. of Science and Tech. (2001), retrieved from www.diva-portal.org/smash/record.jsf?pid=diva2:123855 (viewed 08/08/2014).

21 M. Poletti, "An investigation of 2-d multizone surround sound systems", in Proc. 125th AES Conv., San Francisco, 2-5 October 2008.

22 M. Olik, P. J. B. Jackson, and P. Coleman, "Influence of low-order room reflections on sound zone system performance", in Proc. ICA2013, Montreal, 2-7 June 2013.

23 L. D. Fielder, "Analysis of traditional and reverberation-reducing methods of room equal- 
ization", J. Audio Eng. Soc. 51, 3-26 (2003).

24 P. A. Nelson and S. J. Elliott, Active Control of Sound, pp. 356-378 (Academic Press Limited, London) (1992).

25 S. J. Elliott and P. A. Nelson, "Multiple-point equalization in a room using adaptive digital filters", J. Audio Eng. Soc. 37, 899-907 (1989).

26 B. D. Radlović, R. C. Williamson, and R. A. Kennedy, "Equalization in an acoustic reverberant environment: robustness results", IEEE Trans. Speech Audio Proc. 8, 311$319(2000)$.

27 S. J. Elliott and J. Garcia-Bonito, "Active cancellation of pressure and pressure gradient in a diffuse sound field", J. Sound Vib. 186, 696-704 (1995).

28 F. Asano, Y. Suzuki, and T. Sone, "Sound equalization using derivative constraints", Acta Acust. united Ac. 82, 311-320 (1996).

29 W.-K. Tseng, B. Rafaely, and S. J. Elliott, "Local active sound control using 2-norm and œ-norm pressure minimization", J. Sound Vib. 234, 427-439 (2000).

30 P. D. Hatziantoniou and J. N. Mourjopoulos, "Real-time room equalization based on complex smoothing: robustness results", in Proc. AES 116th Conv., Berlin, 8-11 May 2004.

31 K. Guo and J. Pan, "Effects of reflective ground on the actively created quiet zones", J. Acoust. Soc. Am. 103, 944-952 (1998).

32 M. A. Akeroyd, J. Chambers, D. Bullock, A. R. Palmer, A. Q. Summerfield, P. A. Nelson, and S. Gatehouse, "The binaural performance of a cross-talk cancellation system with matched or mismatched setup and playback", J. Acoust. Soc. Am. 121, 1056-1069 (2007).

33 M. Buck, "Aspects of first-order differential microphone arrays in the presence of sensor imperfections", Europ. Trans. Telecom. 13, 115-122 (2002).

34 P. S. Ow and T. E. Morton, "Filtered beam search in scheduling", Int. J. Prod. Res. 6, 35-62 (1988).

35 J. Francombe, R. Mason, M. Dewhirst, and S. Bech, "Modelling listener distraction resulting from audio-on-audio interference", in Proc. ICA2013, Montreal, 2-7 June 2013. 
TABLE I: Comparisons of sound zone systems under anechoic and reflective conditions reported in the literature. Contrast values are based on figure readings. In all examples, the acoustic contrast control method was used.

\begin{tabular}{|c|c|c|c|c|}
\hline Study & Source array & Frequency range & $\begin{array}{c}\text { Acoustic } \\
\text { environment }\end{array}$ & $\begin{array}{l}\text { Acoustic } \\
\text { contrast }\end{array}$ \\
\hline $\begin{array}{c}\text { Elliott and } \\
\text { Jones }^{4}\end{array}$ & $\begin{array}{c}\text { Two loudspeakers } \\
\text { face to face }\end{array}$ & $0.05-1 \mathrm{kHz}$ & $\begin{array}{l}\text { Anechoic room } \\
\text { Reflective room }\end{array}$ & $\begin{array}{l}>28 \mathrm{~dB} \\
>22 \mathrm{~dB}\end{array}$ \\
\hline $\begin{array}{c}\text { Jacobsen } \\
\text { et al. }{ }^{9}\end{array}$ & $\begin{array}{l}\text { Sixteen point sources } \\
\text { around zones }\end{array}$ & $0.1-1 \mathrm{kHz}$ & $\begin{array}{l}\text { Simulated anechoic } \\
\text { Simulated reflective }\end{array}$ & $\begin{array}{l}>27 \mathrm{~dB} \\
>10 \mathrm{~dB}\end{array}$ \\
\hline $\begin{array}{c}\text { Elliott et } \\
a{ }^{13}{ }^{13}\end{array}$ & $\begin{array}{c}\text { Three point sources } \\
\text { in a line }\end{array}$ & $0.1-2 \mathrm{kHz}$ & $\begin{array}{l}\text { Simulated anechoic } \\
\text { Simulated diffuse } \\
\text { (20\% influence) }\end{array}$ & $>9 \mathrm{~dB}$ \\
\hline Cheer ${ }^{10}$ & $\begin{array}{l}\text { Eight point sources } \\
\text { forming four compact } \\
\operatorname{arrays}^{a}\end{array}$ & $0.1-0.7 \mathrm{kHz}$ & $\begin{array}{c}\text { Simulated anechoic } \\
\text { Simulated } \\
\text { reflective }\end{array}$ & $>30 \mathrm{~dB}$ \\
\hline
\end{tabular}

${ }^{a}$ Example configuration. Similar performance trends were observed for other source arrangements at low frequencies. 
TABLE II: Components of the squared sound pressure at the $n$th monitor sensor in zone B, generated by a $2 \times 2$ pressure canceler. Superscripts $D, R$, and ' indicate relationship with the direct sound canceler, the reflection canceler, and the reflected sound respectively.

\begin{tabular}{l}
\hline$\hat{O}_{B n}^{D}=\sin \left(k d\left(\cos \theta_{B n}-\cos \phi_{B}\right) / 2\right) / s_{B n} r_{B}$ \\
$\hat{O}_{B n}^{D^{\prime}}=\gamma \sin \left(k d\left(\cos \theta_{B n}^{\prime}-\cos \phi_{B}\right) / 2\right) / s_{B n}^{\prime} r_{B}$ \\
$\hat{O}_{B n}^{R}=\gamma \sin \left(k d\left(\cos \theta_{B n}-\cos \phi_{B}^{\prime}\right) / 2\right) / s_{B n} r_{B}^{\prime}$ \\
$\hat{O}_{B n}^{R^{\prime}}=\gamma^{2} \sin \left(k d\left(\cos \theta_{B n}^{\prime}-\cos \phi_{B}^{\prime}\right) / 2\right) / s_{B n}^{\prime} r_{B}^{\prime}$ \\
$\hat{O}_{B n}^{D D^{\prime}}=2 \hat{O}_{B n}^{D} \hat{O}_{B n}^{D^{\prime}} \cos \left(k\left(s_{B n}-s_{B n}^{\prime}\right)\right)$ \\
$\hat{O}_{B n}^{D R}=2 \hat{O}_{B n}^{D} \hat{O}_{B n}^{R} \cos \left(k\left(r_{B}-r_{B}^{\prime}\right)\right)$ \\
$\hat{O}_{B n}^{D R^{\prime}}=2 \hat{O}_{B n}^{D} \hat{O}_{B n}^{R^{\prime}} \cos \left(k\left(s_{B n}-s_{B n}^{\prime}+r_{B}-r_{B}^{\prime}\right)\right)$ \\
$\hat{O}_{B n}^{D^{\prime} R}=2 \hat{O}_{B n}^{D^{\prime}} \hat{O}_{B n}^{R} \cos \left(k\left(s_{B n}-s_{B n}^{\prime}-r_{B}+r_{B}^{\prime}\right)\right)$ \\
$\hat{O}_{B n}^{D^{\prime} R^{\prime}}=2 \hat{O}_{B n}^{D^{\prime}} \hat{O}_{B n}^{R^{\prime}} \cos \left(k\left(r_{B}-r_{B}^{\prime}\right)\right)$ \\
$\hat{O}_{B n}^{R R^{\prime}}=2 \hat{O}_{B n}^{R} \hat{O}_{B n}^{R^{\prime}} \cos \left(k\left(s_{B n}-s_{B n}^{\prime}\right)\right)$ \\
\hline \hline
\end{tabular}


TABLE III: Frequency averaged acoustic contrast produced by systems based on the ACC and SPM methods. A and R denote anechoic and reflective conditions at source position optimization - source weight calculation - sound field evaluation stages respectively, and M represents the median value.

\begin{tabular}{ccccc}
\hline \multirow{2}{*}{ Surface } & Method & \multicolumn{3}{c}{ Contrast (dB) } \\
& & $2 \times 2$ & $2 \times 50$ & $3 \times 50$ \\
\hline None & ACC A-A-A & 54.6 & 56.4 & 93.9 \\
\hline \multirow{4}{*}{ North } & ACC M-R-R & 4.0 & 7.4 & 26.0 \\
& ACC A-A-R & 16.6 & 16.6 & 20.9 \\
& ACC A-R-R & 14.6 & 17.6 & 53.8 \\
& ACC R-R-R & $\mathbf{3 3 . 3}$ & $\mathbf{3 4 . 5}$ & $\mathbf{7 4 . 6}$ \\
& APM R-R-R & 12.1 & 15.8 & 19.6 \\
\hline \multirow{4}{*}{ East } & ACC M-R-R & 1.4 & 3.4 & 19.0 \\
& ACC A-A-R & 38.2 & 38.4 & 62.1 \\
& ACC A-R-R & 34.4 & 38.7 & 79.5 \\
& APM R-R-R & 15.9 & 16.3 & 18.6 \\
\hline \multirow{2}{*}{ West } & ACC R-R-R & $\mathbf{2 0 . 5}$ & $\mathbf{2 3 . 6}$ & $\mathbf{3 0 . 0}$ \\
\hline \hline \multirow{2}{*}{ East, } & ACC M-R-R & 5.0 & 7.6 & 11.3 \\
& ACC A-R-R & 14.3 & 17.6 & 46.4 \\
& ACC A-A-R & 12.5 & 12.5 & 12.6 \\
West & ACC A-R-R & 16.2 & 16.8 & 38.5 \\
& ACC R-R-R & $\mathbf{3 5 . 3}$ & $\mathbf{3 8 . 3}$ & $\mathbf{6 2 . 2}$ \\
& APM R-R-R & 13.1 & 13.4 & 13.7 \\
\hline
\end{tabular}




\section{List of Figures}

FIG. 1 A $2 \times 2$ sound zone system. Symbols: $\square$ source, $\square$ image source, - array axis, - setup sensor, o monitor sensor, $\boldsymbol{\square}$ surface. . . . . . . . . . . . . 9

FIG. 2 Source positions for minimizing the reflected sound pressure in zone B. Symbols as in Fig. 1. . . . . . . . . . . . . . . . . . . . . . 13

FIG. 3 Ranges of angles between the array axis and the monitor sensors in zone B, and the related cosine function values for configurations from Fig. 2 (indicated by the filled regions). . . . . . . . . . . . . . . . . . . . . . . 14

FIG. 4 (a) The normalized direct squared sound pressure component at the setup sensor in zone $\mathrm{A},\left(\hat{O}_{A}^{D}\right)^{2}$ (decibel scale with a $-60 \mathrm{~dB}$ limit). Outside the grayed-out region, $10 \log _{10}\left(\left(\hat{O}_{A}^{D}\right)^{2}\right) \geq-6 \mathrm{~dB}$ in at least $2 / 3$ of the frequency range. (b) Ranges of $\theta_{A}$ that increase the contribution of pressure in zone A to contrast (white regions). Arrays corresponding to the marked values in Fig. 6: $\bullet$ Null-Split, $\boldsymbol{\nabla}$ Far-Align, $\bullet$ Near-Align. . . . . . . . . . . . . 17

FIG. 5 A $2 \times 2$ system with a surface in a number of locations. The surface is tangent to the semi-circle at each angular position indicated by the arrowhead Remaining symbols: $\square$ zone, $\times$ source array center: Far- and Near-Align, $\otimes$ source array center: Null-Split, — example array axis, - example surface; in close-up: • setup sensor, o monitor sensor. . . . . . . . . . . . . . . . . . 19

FIG. 6 Frequency averaged acoustic contrast produced by the Null-Split, Far-Align, and Near-Align arrays with a surface in different positions, as shown in Fig. 5. The arrays producing the marked contrast values are indicated in Fig. 4b, thus the thick portions of the curves correspond to arrangements that increase the contribution of pressure in zone A to contrast. . . . . . . . . . 20

FIG. 7 Configuration used in the numerical search for optimal source positions. Sym-

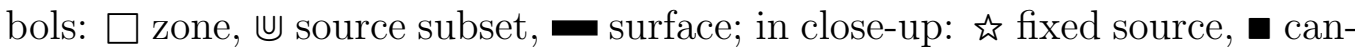
didate source. . . . . . . . . . . . . . . . . . . . . . 2 
FIG. 8 Optimized source arrays for a $2 \times 2$ system with a single surface in different locations: (a) North, (b) East and (c) West, and the sound pressure level maps at $1 \mathrm{kHz}$. Symbols: $\square$ zone, $\times$ array center, - array axis, - surface. . 22

FIG. 9 Optimized source arrays with surfaces generating first order reflections, and the sound pressure level maps at $1 \mathrm{kHz}$ : (a) $2 \times 50$, North; (b) $3 \times 50$, North; (c) $2 \times 2$, East and North; and (d) $2 \times 2$, East and West surface locations. Symbols

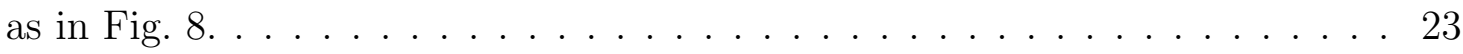

